Fetal Diagnosis and Therapy

\title{
Incidence of Cerebral Injury in Monochorionic Twin Survivors after Spontaneous Single Demise: Long-Term Outcome of a Large Cohort
}

\author{
Mariano Matteo Lanna ${ }^{a}$ Dario Consonni ${ }^{b}$ Stefano Faiola ${ }^{a}$ Giana Izzo ${ }^{c}$ \\ Barbara Scelsa $^{d}$ Irene Cetin $^{\text {e }}$ Maria Angela Rustico ${ }^{a}$ \\ ${ }^{a}$ Fetal therapy Unit "U. Nicolini," Vittore Buzzi Children's Hospital, Università di Milano, Milan, Italy; ${ }^{\text {b Unit of }}$ \\ Epidemiology, Fondazione IRCCS Ca'Granda - Ospedale Maggiore Policlinico, Milan, Italy; ' Department of Pediatric \\ Radiology and Neuroradiology, Buzzi Children's Hospital, Milan, Italy; ${ }^{d}$ Unit of Pediatric Neurology, Buzzi Children's \\ Hospital, Milan, Italy; ${ }^{e}$ Department of Obstetrics and Gynecology, Vittore Buzzi Children's Hospital, Università di \\ Milano, Milan, Italy
}

\section{Keywords}

Monochorionic twins · Cerebral injury · Single fetal demise ·

Twin-to-twin transfusion syndrome

\begin{abstract}
Objectives: To evaluate incidence of cerebral injury and outcome in a large series of monochorionic (MC) twin survivors after spontaneous single fetal demise. Methods: Retrospective analysis of all MC pregnancies with single fetal demise diagnosed at, or referred to, the Fetal Therapy Unit "U. Nicolini," V. Buzzi Children's Hospital, Milan, Italy, from 2004 to 2015. Survivors evaluation protocol included detailed ultrasound (US) of intracranial anatomy, Doppler investigation of peak systolic velocity in the middle cerebral artery (MCA-PSV), and magnetic resonance (MR). Data were collected on pregnancy characteristics, postnatal brain scan, and MR and neurological follow-up. Results: Seventy-eight consecutive MC pregnancies were analyzed. Median gestational age (GA) at single fetal demise was 22 weeks (range 15-36); median interval between single demise and live birth was 105 days (range 1-175), with a median GA at birth of 36 weeks (range 23-41). Prenatal MR was performed in 57 of 78 cases (73\%). Cerebral injury affected $14 / 78$ (18\%) co-twins, 2 of whom were born immediately after single demise, with postnatal diagnosis of cerebral injury; of
\end{abstract}

the other 12 fetuses that were studied before birth, 10 had a prenatal diagnosis of lesion both with US and MR, one only with $M R$, and in one case, a grade III intraventricular hemorrhage was reported only after delivery, which occurred at 25 weeks, 5 weeks after the single demise. Signs of fetal anemia (MCA-PSV value above 1.55 MoM) were related to a higher risk of prenatal cerebral injury; cases with postnatal diagnosis of lesion were delivered at lower GA. Conclusions: Cerebral injury affects $18 \%$ of co-twin survivors after single fetal demise in MC twin pregnancies, and evaluation and follow-up of these cases can improve detection rate of such damage.

(c) 2019 S. Karger AG, Basel

\section{Introduction}

Monochorionic (MC) twins are at increased risk of in utero fetal death (IUD), as a consequence of typical complications such as twin-to-twin transfusion syndrome (TTTS) and selective intrauterine growth restriction (sIUGR), or even in absence of any clear cause [1]. Single fetal demise exposes the co-twin survivor to exsanguination in the empty placental territory through vascular anastomoses, with subsequent acute hypovolemia [2, 3], which is responsible for multi-organ damage, including

\section{KARGER}

(c) 2019 S. Karger AG, Basel 
cerebral injury, or death. It is assumed that the risk of cerebral injury in MC twin survivors after single IUD is four times higher than in dichorionic ones [4]. A protective role against cerebral injury in survivor $\mathrm{MC}$ twins has been identified in laser coagulation of placental anastomoses as treatment of TTTS $[5,6]$ and in cord occlusion of the twin at high risk of death as in cases of severe sIUGR $[7,8]$.

A systematic review and meta-analysis on single fetal demise has revealed a significant heterogeneity in methods of assessment of adverse outcome for surviving twins, with small series reported in various studies [9].

In this study, there is an evaluation of a single-center series with a large number of cases managed by the same operators over a long period of observation. The aim of this study was (1) to evaluate the incidence of cerebral injury in a large series of single twin survivors after the spontaneous death of one twin, occurring in complicated or uncomplicated MC pregnancies, (2) to assess which risk factors are detectable with ultrasound (US) and might therefore influence prenatal counseling, and (3) to evaluate the outcome in this group of single survivors.

\section{Methods}

This is a retrospective analysis of all consecutive MC twin pregnancies with single twin demise observed at, or referred to, the "U. Nicolini” Fetal Therapy Unit of the Vittore Buzzi Children's Hospital, Milan, between January 2004 and December 2015. Cases with fetal demise of the co-twin within the subsequent $48 \mathrm{~h}$ and cases in which fetal death occurred in the first trimester were excluded. Pregnancies complicated with TTTS treated with endoscopic laser coagulation of placental anastomoses and cases of selective feticide performed with bipolar cord coagulation were also excluded. Cases with fetal anomalies were included unless they had undergone selective feticide or laser for TTTS. TTTS was defined by polyhydramnios-oligohydramnios sequence according to EUROFETUS criteria for maximum vertical pocket in recipient and donor $(\geq 8$ $\mathrm{cm}$ before 20 weeks of gestational age [GA] and $\geq 10 \mathrm{~cm}$ after 20 weeks GA and $<2 \mathrm{~cm}$, respectively) [5]; sIUGR was defined by the presence of one twin with estimated fetal weight $<10$ th percentile and inter-twin estimated fetal weight discordance $>25 \%[10]$.

In each case, the following data were recorded: maternal age, parity, amnionicity, presence of TTTS, sIUGR, referred with a single fetal demise before a treatment option was possible, and GA at detection of single fetal death. In order to search for signs of fetal anemia, Doppler measurement of the middle cerebral artery peak systolic velocity (MCA-PSV) was performed as soon as the event was detected and no longer than 7 days after the event [11]. Serial two-dimensional and three-dimensional US was performed to evaluate the fetal brain in surviving twins, performed transabdominally (in breech presentation) and/or transvaginally (GE E8, GE Voluson 730, Medical Systems, Zipf, Austria).

Magnetic resonance (MR) was offered to the parents and scheduled at least 2 weeks after single fetal demise or within a few days in presence of suspected fetal brain injury at US, as already described and reported in previous series $[12,13]$. MR was performed on a 1.5-T system (Achieva-Dual, Philips Healthcare, Eindhoven, The Netherlands) with a phased array abdominal coil and with a protocol including T2-weighted single-shot FSE multiplanar sections (3- to 4-mm-thick sections; gap, $0.1 \mathrm{~mm}$; TR/TE, 3,000/180 ms; in-plane resolution, $1.1 \mathrm{~mm}^{2}$ ), balanced steady-state multiplanar sections (contiguous 2- to 3-mm-thick sections), T1weighted FSE multiplanar sections (5.5-mm-thick sections; TR/ TE, 300/14 ms; turbo factor, 3; in-plane resolution, $1.4 \mathrm{~mm}^{2}$ ), and in several cases, DWI sections (5.5-mm-thick sections; TR/TE, $1,000 / 90 \mathrm{~ms}$; b factor, $0-600 \mathrm{~s} / \mathrm{mm}^{2}$; matrix, $\left.128 \times 128\right)$ and FLAIR fat sat sections (4.0 mm thickness; TR/TE, 6,000/54 ms; TI, 2,000 $\mathrm{ms}$; turbo factor, 63; in-plane resolution, $1.25 \mathrm{~mm}^{2}$ ).

In continuing pregnancies, fetal growth assessment, MCAPSV, and umbilical artery Doppler were scheduled every 2 weeks. Data on pregnancy outcome, GA at birth, and birth weight were also recorded. All live-born neonates underwent an early brain scan and, in selected cases of suspected cerebral injury, MR in the first 28 days of life.

The definition of cerebral injury included cases detected both by prenatal and by postnatal US and MR. US findings that defined cerebral injury were: irregular shape of lateral ventricles, ventriculomegaly, cortical hyperecogenicity, periventricular cysts, and encephalomalacia.

All surviving infants were followed up with serial neurological examinations by a pediatric neurologist-psychiatrist, which is routine practice in Italy. Postnatal follow-up ranged from 2 to 7 years (median, 36 months) and was recorded for all neonates/infants.

The Griffiths Mental Scale Developmental Test was used to assess cognitive and motor development. Neurological impairments were classified as severe, moderate, or mild [14]. Patients classified as having severe impairment were those with motor deficits impairing their ability to walk (cerebral palsy level 3-5), development quotient $<70$, severe behavioral disorder (autism), or bilateral sensorineural deficit (bilateral deafness or blindness). Children classified as having moderate disability were those with motor deficits not impairing their ability to walk (cerebral palsy level 2), development quotient of 70-84, behavioral disorders (attention deficit and/or hyperactivity), or unilateral sensorineural deficit. Children classified as having mild disability were those with minor motor deficit (clumsiness), transient motor delay (with prospect of normalization), or isolated language impairment [15]. The study complied with our Institution's research guidelines for clinical observational studies.

\section{Statistical Analysis}

We fitted univariate logistic regression models to calculate odds ratios (OR) and 95\% confidence intervals (CI) of cerebral injury according to selected variables, such as type of complication, signs of fetal anemia, and GA at the event and at birth. Statistical analyses were performed with Stata 14 (StataCorp 2015).

\section{Results}

A total of $78 \mathrm{MC}$ pregnancies with single twin demise were included in the study. Table 1 describes the antenatal characteristics at the time of referral. Median GA at the 
Fig. 1. Flowchart.

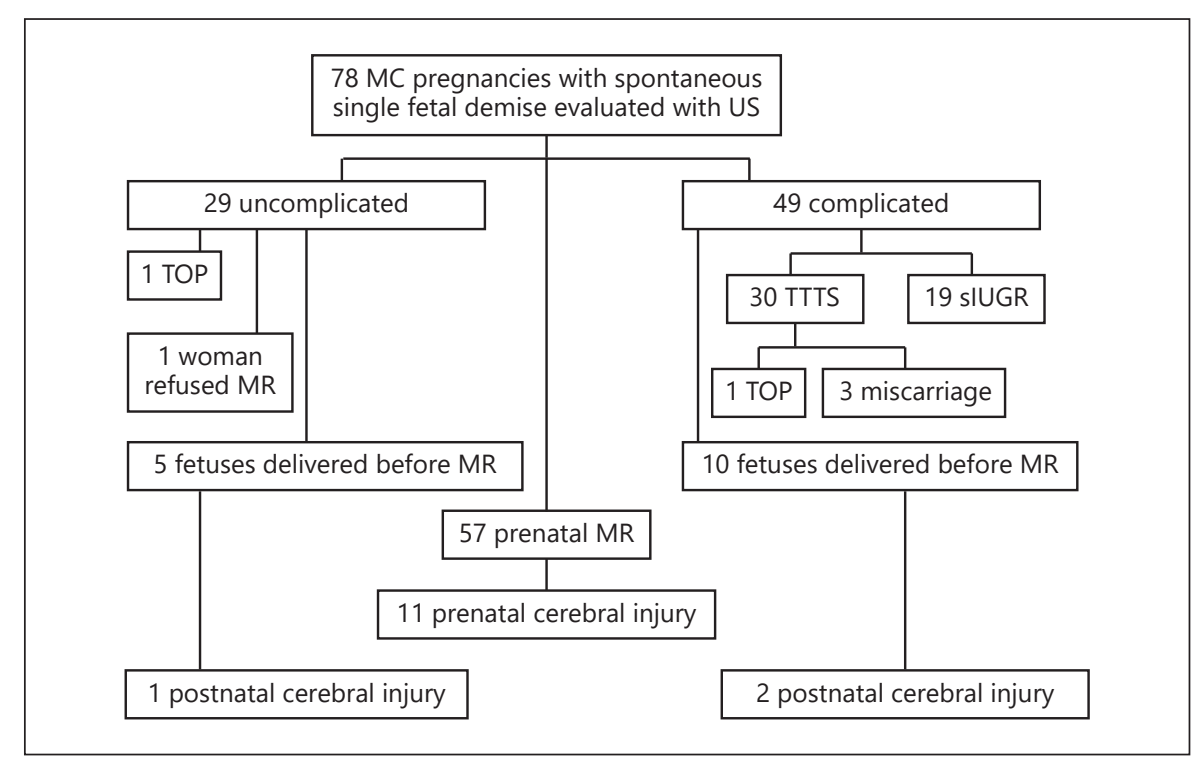

time of detection of single fetal demise was 22 weeks (15$36)$. Forty-nine (63\%) cases occurred in complicated MC pregnancies, more frequently in TTTS $(30 / 78,38 \%)$ than in MC pregnancies with sIUGR (19/78, 24\%). In 29 cases (37\%), the single demise occurred unexpectedly during the US surveillance scheduled every 2 weeks without any evidence of complication. A sign of fetal anemia, with MCA-PSV Doppler $>1.55$ multiple of median, was detected in $11(14 \%)$ survivors, two of whom received an intrauterine fetal transfusion (IUT), performed after parents were counseled and gave their consent to the procedure.

Prenatal MR was performed in 57 cases (73\%) (see Fig. 1 for details). In none of the 21 cases without prenatal MR were there US findings of cerebral injury. Table 2 shows the characteristics and outcome of the cases with cerebral injury, that occurred overall in 14 cases (18\%). Of the 11 cases detected before birth, US identified 10 cases.

Termination of pregnancy was chosen in 9 of the 11 twins and fetal autopsy confirmed prenatal findings in all cases (examples in Fig. 2-5).

Of the two surviving neonates with a prenatal detection of cerebral injury (cases 1 and 2), long-term followup revealed severe and mild neurological impairment, respectively, described in Table 2. Cerebral injury was diagnosed only postnatally (cases 13 and 14) in two cases where the operator decided for immediate delivery of the survivor, following diagnosis of single demise. In the third case of postnatal diagnosis of cerebral injury (case 12 ), severe prematurity ( 25 weeks of GA) was the cause of
Table 1. Antenatal characteristics and perinatal outcome of 78 monochorionic (MC) pregnancies complicated with single fetal demise

\begin{tabular}{lc}
\hline Characteristics & $N=78$ MC pregnancies \\
\hline GA at IUD, weeks & $22(15-36)$ \\
Without complication & $29(37)$ \\
With complication & $49(63)$ \\
$\quad$ TTTS & $30(38)$ \\
$\quad$ sIUGR & $19(24)$ \\
Sign of anemia in co-twin & $11(14)$ \\
IUT & $2(2)$ \\
Prenatal MR & $57(73)$ \\
GA at MR, weeks & $23.9(20.3-33.6)$ \\
Cerebral injury & $14(18)$ \\
Prenatal US/MR & $11(14)$ \\
Postnatal US/MR & $3(4)$ \\
TOP & $9(11)$ \\
Miscarriage & $3(4)$ \\
IUD $>48$ h & $1(1)^{9}$ \\
Interval demise-delivery, days & $105(1-175)$ \\
GA at delivery, weeks & $36(23-41)$ \\
Birth weight, g & $2,420(500-3,800)$ \\
NND & $3(4)$ \\
\hline
\end{tabular}

Values are indicated with number and percentage in brackets, or with median and range in brackets. GA, gestational age; IUD, in utero fetal death; TTTS, twin-twin transfusion syndrome; sIUGR, selective intrauterine growth restriction; IUT, intrauterine transfusion; US, ultrasound; MR, magnetic resonance; TOP, termination of pregnancy; NND, neonatal death. * All cases of fetal demise were IUGR. ${ }^{\S}$ All cases of cerebral injury detected by US or MR, both prenatal and postnatal. 'This fetus developed edema and cardiac failure as a consequence of a syndrome.
Lanna/Consonni/Faiola/Izzo/Scelsa/ Cetin/Rustico 


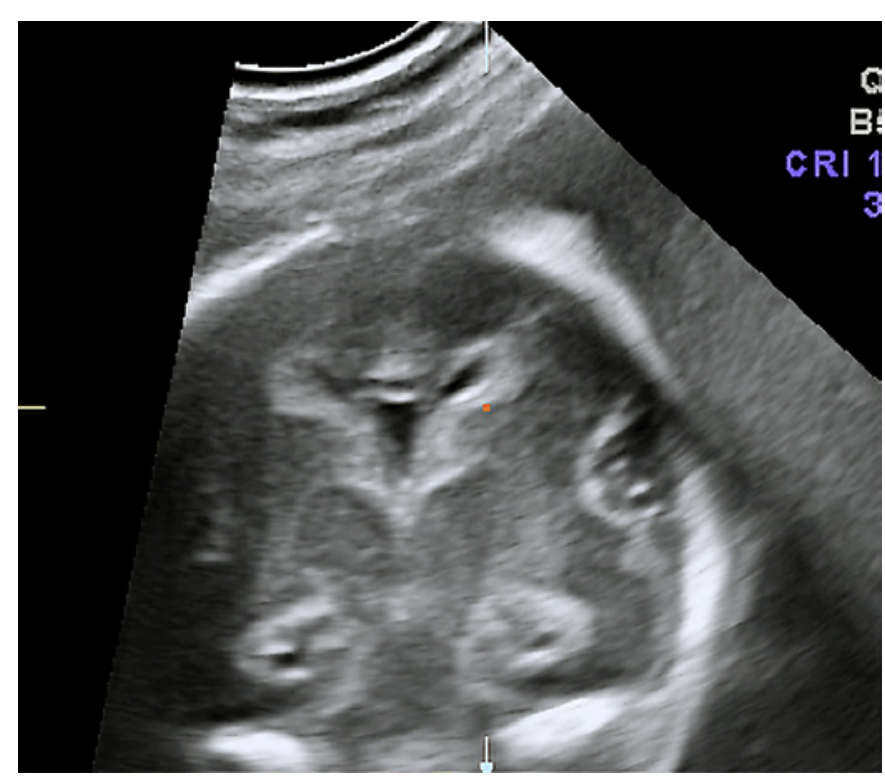

Fig. 2. Cortical hyperechogenicity in a recipient survivor at 21 weeks' gestational age, after spontaneous death of the donor twin (case No. 3 in Table 2).

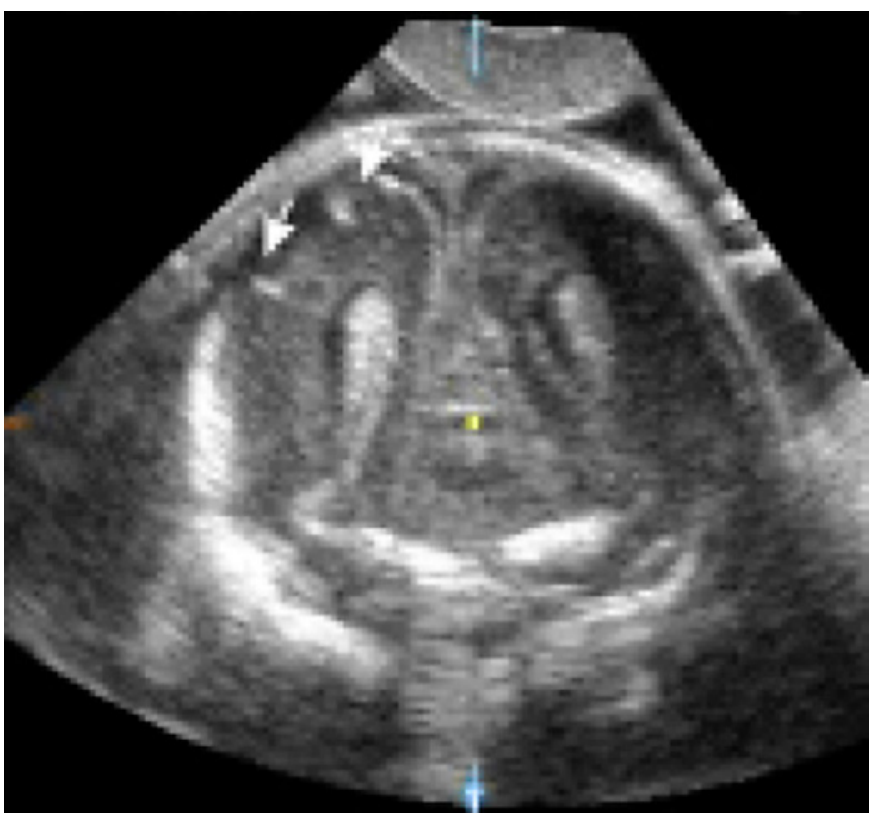

Fig. 4. Cortical lesions in a single survivor at 24 weeks' gestational age, after spontaneous death of the uncomplicated twin (case No. 7 in Table 2).

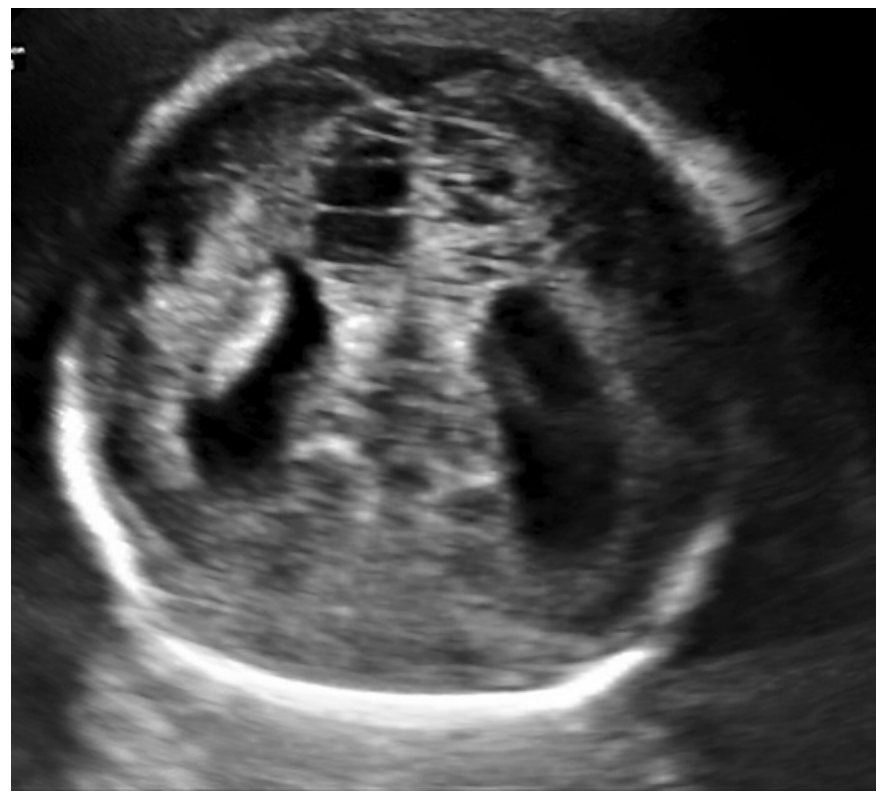

Fig. 3. Periventricular leukomalacia in a single survivor at 24.5 weeks' gestational age, after spontaneous death of the sIUGR twin (case No. 4 in Table 2).

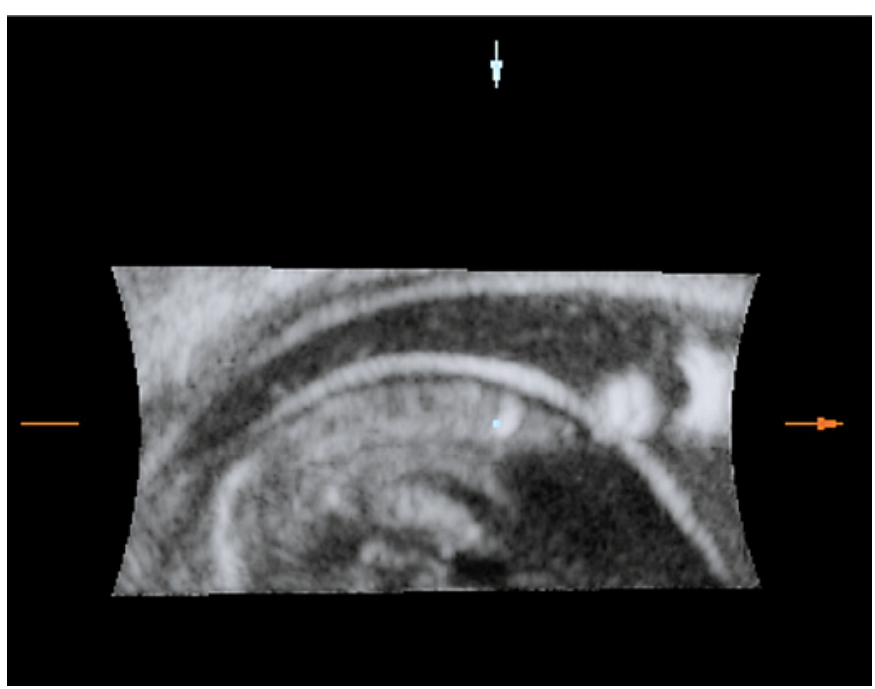

Fig. 5. Cortical lesions in a single survivor at 19.6 weeks' gestational age, after spontaneous death of the uncomplicated twin (case No. 8 in Table 2). 
Table 2. Characteristics of 14 co-twins with cerebral injury after single fetal demise

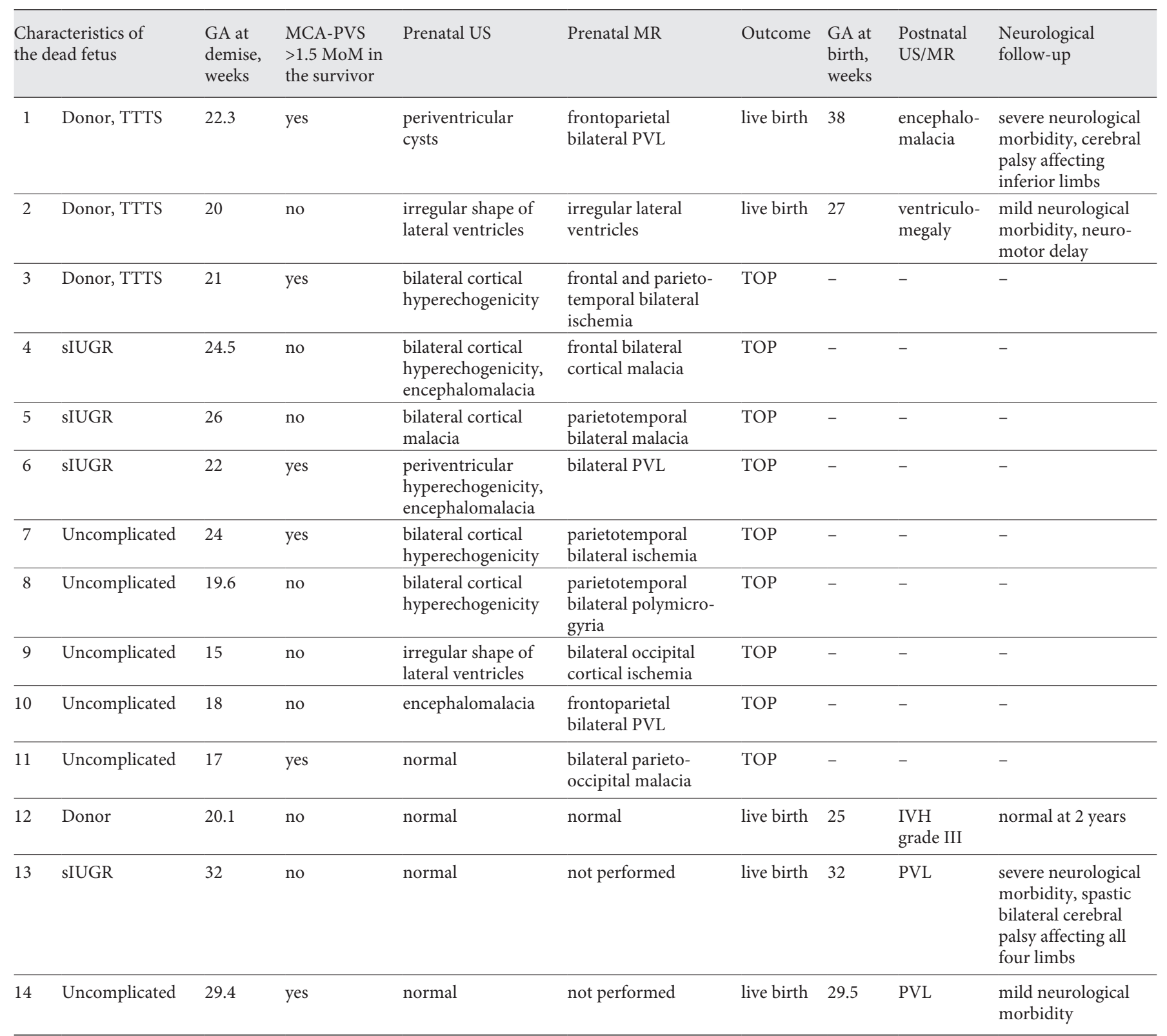

GA, gestational age; MCA-PSV, peak systolic velocity in middle cerebral artery; US, ultrasound; MR, magnetic resonance; TOP, termination of pregnancy; sIUGR, selective intrauterine growth restriction: all were growth-restricted fetuses; PVL, periventricular leukomalacia; IVH, intraventricular hemorrhage; TTTS, twin-twin transfusion syndrome. In cases 12,13 , and 14, the cerebral injury was detected after birth.

a grade III intraventricular hemorrhage that left no consequences at neurological follow-up. Overall, 5 out 11 of the anemic fetuses had cerebral injury: none of these 5 cases had received IUT.

There was a five-fold increased risk of cerebral injury for fetuses with a Doppler value of MCA-PSV suggestive of fetal anemia (Table 3).
A subgroup analysis based on GA at initial IUD and at delivery found that lower GA at IUD correlated with risk of prenatal cerebral injury (OR 0.9, CI 0.87-1.1), while higher GA at delivery correlated with a lower risk of postnatal cerebral injury (OR 0.80 per week, CI $0.66-$ $0.99)$. 
Table 3. Risk factors for cerebral injury in survivors

\begin{tabular}{lccc}
\hline Variable & $\begin{array}{l}\text { No cerebral injury } \\
(n=64)\end{array}$ & $\begin{array}{l}\text { Cerebral injury } \\
(n=14)\end{array}$ & OR (95\% CI) \\
\hline Uncomplicated pregnancy $(n=29)$ & $23(36)$ & $6(43)$ & 1.00 (reference) \\
TTTS $(n=30)$ & $26(40)$ & $4(28)$ & $0.58(0.14-2.35)$ \\
IUGR $(n=19)$ & $15(23)$ & $4(28)$ & $1.02(0.24-4.23)$ \\
MCA-PSV $>1.55$ MoM & $6(9)$ & $22(19.6-24.5)$ & $0.98(0.87-1.10)$ \\
GA at IUD & $22(19.4-25.4)$ & $29.6(27-32)$ & $0.80(0.66-0.98)$ \\
GA at birth & $36.1(34-38)$ & \\
\hline
\end{tabular}

Values are indicated with number and percentage in brackets, or with median and interquartile range in brackets. OR, odds ratio, from univariate logistic regression; CI, confidence interval; GA, gestational age; IUD, intrauterine fetal death; TTTS, twin-twin transfusion syndrome; sIUGR, selective intrauterine growth restriction; MoM, multiple of median; MCA-PSV, middle cerebral artery peak systolic velocity.

\section{Discussion}

This is the largest series ever described of single survivors after spontaneous fetal demise in MC twin pregnancies. The strength of this retrospective study is its high percentage of cases studied with US and then confirmed by MR findings (73\%), which helps to reduce bias in the evaluation of prognostic factors. In a previous series of 50 fetuses by van Klink et al. [16], MR was performed in order to confirm US findings in $44 \%$ of cases, making the identification of the cases with cerebral injury less accurate. In our series, there was only one case of discordance between a negative prenatal imaging and a positive postnatal US/MR for cerebral injury, namely a grade III intraventricular hemorrhage due to severe prematurity. The three cases in which the single survivor was delivered immediately before any examination with dedicated US or MR could be performed, occurred at early GA $(29,29$, and 32 weeks), which may have contributed to the development of cerebral injury.

In this series, all cases with prenatal evidence of cerebral injury were studied by dedicated US, which identified the presence of a lesion in $91 \%$ of cases, subsequently confirmed with MR. In the only case in which US did not find the lesion evident at MR, there were signs of severe fetal anemia, considered as a risk factor for cerebral injury. Although this study was not powered to demonstrate the accuracy of US, there was no discordance with US in any of the remaining cases, free from injury, which underwent MR. This finding is in contrast with that of Robinson et al. [17], who described a series of complicated MC pregnancies in which prenatal MR was found to change prognostic counseling in $30 \%$ of survivors after spontaneous single demise. In our experience, normal US of the fetal brain was always followed by a concordant $\mathrm{MR}$, with a reassuring counseling for prognosis. This is especially helpful when parents ask for legal termination of pregnancy, and confirmation of US findings with MR cannot be delayed. The number of cases makes it impossible for us to validate this correlation, but it could be a starting point for further studies and a meta-analysis.

Postnatal outcome was available for all cases, with a long-term follow-up (at least 2 years) that made it possible to identify any neurological impairment that might derive from a cerebral injury.

Over two decades ago, fetal anemia was identified as the main cause of brain damage in survivor MC twins [2], and MCA-PSV Doppler velocimetry has become established as the first step with which to identify fetuses at risk [11], since the correlation of fetal anemia with poor outcome of single twin survivor has been proved [18]. An MCA-PSV Doppler velocimetry suggestive of fetal anemia may mean a five-fold increase in the relative risk of cerebral injury. Notwithstanding that, normal values of MCA-PSV are still not sufficient cause for reassuring counseling until MR has been performed, since MCAPSV values may have normalized during the time interval between demise and observation.

It is still unclear whether IUT can reduce the risk in anemic fetuses, and the optimal timing for a total rescue is still being debated. However, some authors recommend it as a first step in the management of anemic survivors [19]. In our series, IUT was performed in only 2 cases, when diagnosis of single demise was made within $24 \mathrm{~h}$, at a GA that enabled parents to decide for legal termination of pregnancy. 
Another important finding of this study is that even in so-called uncomplicated MC pregnancies, for which the 2 -week interval of ultrasonographic follow-up was assumed as optimal to identify complications, the wellknown risk of unexpected fetal demise [1] carries a $20 \%$ risk of cerebral injury, a percentage in line with what was reported in a recent review [9], and just as high as in complicated cases, thus supporting the hypothesis that cerebral injury is related to vascular anastomoses and not necessarily to TTTS or sIUGR. There is no valid explanation for the cases free from injury, while the evidence for different types of lesion was found to correlate with the type of complication [13].

In uncomplicated twin pregnancies, the finding of a single demise makes counselling the parents more challenging, since no explanation can be offered regarding the presumed cause of death, and no treatment can prevent the damage of the survivor.

In such cases, knowledge of the prognostic factors for cerebral injury can be very helpful to operators. Immediate delivery is not recommended as a preventive measure for cerebral injury [7], while the best option has been proven to be waiting for the prognosis of the survivor to be defined by means of MR [17]: this is a limitation of our study since in some cases, delivery occurred before adequate examination.

Multivariate analysis showed no association with type of complication and cerebral injury, unlike the findings in previous series [16], where TTTS was identified as the leading cause of cerebral injury.

In our sIUGR cases, all survivors were larger twins of MC pregnancies included in a recent reported series [20], in which systematic follow-up was performed: no explanation has been found for the severity of damage to survivors.
As reported in the results, low GA at birth was a positive predictor for postnatal cerebral injury, in line with findings in the series described by van Klink et al. [16].

In conclusion, single fetal demise in MC twins is a serious event that can occur even in the absence of any complication. Survivors are exposed to a risk of cerebral injury that can be diagnosed with both US and MR and evaluated by a pediatric neurologist as long as the patient is referred to a tertiary center. In Italy, legal time limits for termination of pregnancy make it crucial for parents to be informed about the prognosis for the fetus as early as possible. This is a limitation of our study since many cases with a lesion could not have further assessment in gestation. Clearly, whenever surviving fetuses are found to be free of cerebral injury, the risk of a poor postnatal outcome is significantly lower if delivery occurs at an advanced GA. Long-term follow-up did not reveal any discordance between expectation after in utero evaluation and postnatal clinical outcome, so long as proper evaluation is performed before birth.

The absence of signs of fetal anemia and normal neuro-sonography may provide parents with enough reassurance to wait for an adequate GA for prenatal MR investigation.

\section{Statement of Ethics}

The authors have no ethical conflicts to disclose.

\section{Disclosure Statement}

The authors have no conflicts of interest to declare.

\section{References}

1 Sebire NJ, Snijders RJ, Hughes K, Sepulveda W, Nicolaides KH. The hidden mortality of monochorionic twin pregnancies. Br J Obstet Gynaecol. 1997 Oct;104(10):1203-7.

2 Nicolini U, Poblete A. Single intrauterine death in monochorionic twin pregnancies. Ultrasound Obstet Gynecol. 1999 Nov;14(5): 297-301.

3 Argoti PS, Bebbington MW, Johnson A, Moise KJ Jr. Sonographic capture of acute exsanguination in a case of developing monochorionic cotwin demise. Ultrasound Obstet Gynecol. 2013 Jul;42(1):119-20.
4 Hillman SC, Morris RK, Kilby MD. Co-twin prognosis after single fetal death: a systematic review and meta-analysis. Obstet Gynecol. 2011 Oct;118(4):928-40.

5 Senat MV, Deprest J, Boulvain M, Paupe A, Winer N, Ville Y. Endoscopic laser surgery versus serial amnioreduction for severe twinto-twin transfusion syndrome. N Engl J Med. 2004 Jul;351(2):136-44.

6 Rustico MA, Lanna MM, Faiola S, Schena V, Dell'avanzo M, Mantegazza V, et al. Fetal and maternal complications after selective fetoscopic laser surgery for twin-to-twin transfusion syndrome: a single-center experience. Fetal Diagn Ther. 2012;31(3):170-8.
7 O'Donoghue K, Rutherford MA, Engineer N, Wimalasundera RC, Cowan FM, Fisk NM. Transfusional fetal complications after single intrauterine death in monochorionic multiple pregnancy are reduced but not prevented by vascular occlusion. BJOG. 2009 May; 116(6):804-12.

8 Lanna MM, Rustico MA, Dell'Avanzo M, Schena V, Faiola S, Consonni D, et al. Bipolar cord coagulation for selective feticide in complicated monochorionic twin pregnancies: 118 consecutive cases at a single center. Ultrasound Obstet Gynecol. 2012 Apr;39(4):40713.

Lanna/Consonni/Faiola/Izzo/Scelsa/ Cetin/Rustico
Fetal Diagn Ther 2020;47:66-73

DOI: $10.1159 / 000500774$ 
9 Mackie FL, Rigby A, Morris RK, Kilby MD. Prognosis of the co-twin following spontaneous single intrauterine fetal death in twin pregnancies: a systematic review and metaanalysis. BJOG. 2019 Apr;126(5):569-78.

10 Khalil A, Rodgers M, Baschat A, Bhide A, Gratacos E, Hecher K, et al. ISUOG Practice Guidelines: role of ultrasound in twin pregnancy. Ultrasound Obstet Gynecol. 2016 Feb; 47(2):247-63.

11 Mari G, Detti L, Oz U, Zimmerman R, Duerig P, Stefos T. Accurate prediction of fetal hemoglobin by Doppler ultrasonography. Obstet Gynecol. 2002 Apr;99(4):589-93.

12 Righini A, Salmona S, Bianchini E, Zirpoli S, Moschetta M, Kustermann A, et al. Prenatal magnetic resonance imaging evaluation of ischemic brain lesions in the survivors of monochorionic twin pregnancies: report of 3 cases. J Comput Assist Tomogr. 2004 Jan-Feb; 28(1):87-92.
13 Conte G, Righini A, Griffiths PD, Rustico M, Lanna M, Mackie FL, et al. Brain-injured Survivors of Monochorionic Twin Pregnancies Complicated by Single Intrauterine Death: MR Findings in a Multicenter Study. Radiology. 2018 Aug;288(2):582-90.

14 Marlow N. Measuring neurodevelopmental outcome in neonatal trials: a continuing and increasing challenge. Arch Dis Child Fetal Neonatal Ed. 2013 Nov;98(6):F554-8.

15 Palisano R, Rosenbaum P, Walter S, Russell D, Wood E, Galuppi B. Development and reliability of a system to classify gross motor function in children with cerebral palsy. Dev Med Child Neurol. 1997 Apr;39(4):214-23.

16 van Klink JM, van Steenis A, Steggerda SJ, Genova L, Sueters M, Oepkes D, et al. Single fetal demise in monochorionic pregnancies: incidence and patterns of cerebral injury. Ultrasound Obstet Gynecol. 2015 Mar;45(3): 294-300.
17 Robinson A, Teoh M, Edwards A, Fahey M Goergen S. Fetal brain injury in complicated monochorionic pregnancies: diagnostic yield of prenatal MRI following surveillance ultrasound and influence on prognostic counselling. Prenat Diagn. 2017 Jun;37(6):611-27.

18 Senat MV, Loizeau S, Couderc S, Bernard JP, Ville Y. The value of middle cerebral artery peak systolic velocity in the diagnosis of fetal anemia after intrauterine death of one monochorionic twin. Am J Obstet Gynecol. 2003 Nov;189(5):1320-4.

19 Quarello E, Stirnemann J, Nassar M, Nasr B, Bernard JP, Leleu-Huard F, et al. Outcome of anaemic monochorionic single survivors following early intrauterine rescue transfusion in cases of feto-fetal transfusion syndrome. BJOG. 2008 Apr;115(5):595-601.

20 Rustico MA, Consonni D, Lanna M, Faiola S, Schena V, Scelsa B, et al. Selective intrauterine growth restriction in monochorionic twins: changing patterns in umbilical artery Doppler flow and outcomes. Ultrasound Obstet Gynecol. 2017 Mar;49(3):387-93. 